\title{
Weak momentum scattering and the conductivity of graphene
}

\author{
Dimitrie Culcer and R. Winkler \\ Advanced Photon Source, Argonne National Laboratory, Argonne, IL 60439. and \\ Northern Illinois University, De Kalb, IL 60115.
}

(Dated: October 31, 2018)

\begin{abstract}
Electrical transport in graphene offers a fascinating parallel to spin transport in semiconductors including the spin-Hall effect. In the weak momentum scattering regime the steady-state density matrix contains two contributions, one linear in the carrier number density $n$ and characteristic scattering time $\tau$, the other independent of either. In this paper we take the Liouville equation as our starting point and demonstrate that these two contributions can be identified with pseudospin conservation and non-conservation respectively, and are connected in a non-trivial manner by scattering processes. The scattering term has a distinct form, which is peculiar to graphene and has important consequences in transport. The contribution linear in $\tau$ is analogous to the part of the spin density matrix which yields a steady state spin density, while the contribution independent of $\tau$, is analogous to the part of the spin density matrix which yields a steady state spin current. Unlike in systems with spin-orbit interactions, the $n$ and $\tau$-independent part of the conductivity is reinforced in the weak momentum scattering regime by scattering between the conserved and non-conserved pseudospin distributions.
\end{abstract}

\section{INTRODUCTION}

The zero-gap semiconductor graphene, or twodimensional carbon, is a new material with a host of remarkable physical properties that offers the possibility of all-carbon devices. The last three years have seen a surge of experimental and theoretical interest in graphene following its realization in the laboratory. ${ }^{1-3}$ High-mobility graphene samples are nowadays reliably manufactured, and the recent experimental success in applying a top gate $^{4}$ offers an increased handle on material properties. Among the latest notable successes the ability to suspend graphene experimentally ${ }^{5}$ is expected to help determine the value of the dielectric constant $\epsilon$ unambiguously in this material.

The linear spectrum characterizing the band structure of single-layer graphene is reminiscent of dispersion relations in relativistic physics, and the charge carriers in this material behave like massless Dirac particles. Graphene has a honeycomb lattice with two atoms per unit cell, and the Hamiltonian displays a coupling between electron and hole states which gives rise to a degree of freedom we refer to as the pseudospin. It is these facts that underlie its unusual features, which include the vanishing of the density of states at the Dirac point, a contribution to the conductivity independent of the carrier density $n$ and scattering time $\tau$, a half-integer quantum Hall effect, and the Klein paradox. ${ }^{1-3}$

Recent experimental work includes the fabrication of epitaxial graphene/graphene-oxide junction, ${ }^{6}$ measurement of ultrafast carrier dynamics, ${ }^{7}$ shot noise measurements, ${ }^{8,9}$ determination of the performance limits of graphene devices, ${ }^{10}$ observation of the AharonovBohm effect, ${ }^{11}$ observation of the quantum Hall effect near the Dirac point, ${ }^{12}$ of a renormalization of the velocity due to electron-phonon interaction, ${ }^{13}$ and a thorough experimental study of epitaxial graphene on SiC. ${ }^{14}$ Theoretical research on single-layer graphene has concen- trated on, among other matters, the effect of electronelectron interactions, ${ }^{15}$ which are expected to be important due to weak screening, on the question of whether graphene is a Fermi liquid, ${ }^{16}$ and on the importance of localization around impurities. ${ }^{17} \mathrm{~A}$ number of theories have dealt with scaling, ${ }^{18,19}$ impurity states, ${ }^{20}$ the odd-integer quantum Hall effect, ${ }^{21}$ the fractional quantum Hall effect, ${ }^{22,23}$ polaritons, ${ }^{24}$ spin-orbit coupling ${ }^{25}$ and sum rules for the optical and Hall conductivities. ${ }^{26}$ In addition to these, a large number of theoretical predictions include the quantum spin-Hall effect, ${ }^{27}$ spin-Hall conductance fluctuations, ${ }^{28}$ proximity-induced superconductivity, ${ }^{29}$ antiferromagnetism, ${ }^{30}$ the spin-valve effect, ${ }^{31}$ peculiar focusing properties of graphene $\mathrm{p}-\mathrm{n}$ junctions, ${ }^{32}$ the use of graphene quantum dots as spin qubits, ${ }^{33}$ Weiss oscillations, ${ }^{34}$ and a zero-bias anomaly in the tunneling density of states. ${ }^{35}$ Many other theories have sought to increase theoretical understanding of graphene. ${ }^{36-63} \mathrm{Be}-$ yond the single-layer form, graphene ribbons have been predicted to have spin polarized edge states, ${ }^{64}$ while the band gap in bilayers has been shown to be tunable by means of an electric field. ${ }^{65}$

In the following we shall refer frequently to the weak momentum scattering regime, characterized by $\varepsilon_{F} \gg$ $\hbar / \tau$, where $\tau$ is a characteristic momentum scattering time, and the strong momentum scattering regime in which $\varepsilon_{F} \ll \hbar / \tau$. Furthermore, it is conventional in the literature to make the distinction between intrinsic and extrinsic graphene. Intrinsic graphene refers to the specific case in which the carrier doping density $n$ is zero, and the Fermi energy lies at the Dirac point, $k=0$. Intrinsic graphene is therefore by definition in the strong momentum scattering regime. Extrinsic graphene refers to the doped case and may be in either the weak or the strong momentum scattering regime.

The presence of the additional degree of freedom contained in the pseudospin causes the steady state for graphene in an electric field to be qualitatively different 
from any other known material. In particular, the presence of a contribution to the conductivity independent of the carrier density remains a puzzling observation. Such a contribution has been measured experimentally ${ }^{1-3}$ and we emphasize that it was extracted from the experimental data by taking the doping density to zero. The number obtained is naturally characteristic of the strong momentum scattering regime. It is typically referred to as the minimum conductivity of graphene. At the same time, theoretical research on clean graphene (no scattering) has found an additional contribution to the conductivity independent of $n$ and $\tau \cdot{ }^{36-44}$ There appears to be some agreement that coherence between electrons and holes lies at the heart of this particular contribution to the conductivity. Since the Hamiltonian for carriers in graphene is $\hbar v \boldsymbol{\sigma} \cdot \boldsymbol{k}$, where $v$ is a constant and $\boldsymbol{\sigma}$ represents the pseudospin, the velocity operator is simply $v \boldsymbol{\sigma}$, and it is evident that the pseudospin will play a crucial role in transport. Nevertheless this contribution to the conductivity was found in the ballistic regime, whereas the contribution referred to as the minimum conductivity was measured experimentally in the strong momentum scattering regime. We note that a series of enlightening papers have focused on Boltzmann transport ${ }^{45}$, on transport in the strong momentum scattering limit ${ }^{46,47}$ and transport in extrinsic graphene $e^{48,49}$. Our work differs from these papers in that it is the first to recover both the ordinary $\tau$-dependent "Boltzmann" conductivity and the $n$ - and $\tau$-independent conductivity, and to demonstrate the profound relationship that exists between the two. In addition, our formalism does not require us to work in the limit $n \rightarrow 0$ in order to recover the $n$ - and $\tau$-independent conductivity.

In order to arrive at a tractable equation for the density matrix it is necessary to assume that the Fermi energy $\varepsilon_{F} \gg \hbar / \tau$, which requires us to restrict our attention to the weak momentum scattering regime. We assume low temperatures, where scattering due to charged impurities is important and may be dominant, and the fact that weakly doped graphene is a Fermi liquid ${ }^{16}$ justifies omitting the effect of electron-electron scattering. We believe that our approach sheds light on the manner in which the part of the conductivity of graphene arises that is independent of number density and $\tau$ and how it is related to the ordinary, $\tau$-dependent ("Boltzmann") conductivity. Indeed, the theory presented in this work is tailored towards rendering explicit the important role of the pseudospin in the dynamics of carriers in graphene, with a focus on steady state processes, providing an accurate and at the same time transparent approach. We wish to emphasize that, although we determine the numerical value of the conductivity, our principal aim is not to obtain a number but rather to bring to light the underlying structure of the steady-state density matrix in pseudospin space. Compared to the Boltzmann picture, our formalism has coherence between electrons and holes built in from the start and thus provides a clear physical picture. Nevertheless weak localization effects ${ }^{46,47}$ are not taken into account in this work, as they are not expected to be important in the weak momentum scattering regime.

We find the most important observation to be the fact that a carrier's pseudospin is not conserved, because of electron-hole coherence present in the Hamiltonian (including coherence induced by the electric field). In the absence of intervalley scattering, assumed in our work, a pseudospin eigenstate is an electron or a hole, thus pseudospin non-conservation means a continually changing combination of an electron and a hole. Therefore each carrier can be thought of as a part which is either an electron or a hole, and a part which is a continually changing mixture of an electron and a hole. With this in mind, it makes sense to divide the pseudospin density matrix into two linearly independent contributions, corresponding to conserved pseudospin (the carrier is an electron or a hole) and non-conserved precessing pseudospin (the carrier is a continually changing mixture of an electron and a hole). We show in a systematic fashion that the two contributions to the conductivity of single-layer graphene are related to these linearly independent components of the pseudospin density matrix. The $\tau$-dependent contribution is a result of pseudospin conservation, while the $n$ and $\tau$-independent conductivity stems from pseudospin non-conservation. The two independent parts of the density matrix, often referred to as the dissipative and reactive parts, which are responsible for the two terms in the conductivity, are connected in a non-intuitive way by scattering events. We find that scattering gives rise to a term in the Liouville equation which is peculiar to graphene and distinct from the usual scattering term in other conductors. In particular, even an infinitesimal amount of scattering produces a contribution to zeroth order in the scattering potential which reinforces the $n$ and $\tau$-independent contribution to the conductivity coming from the band structure. This feature is one of the many intriguing analogies which exist between transport in single-layer graphene and the generation of steadystate spin densities and currents, including the spin-Hall effect, in ordinary semiconductors. ${ }^{69,72,73}$ From a different perspective, the two independent parts of the density matrix are intimately related with the phenomenon of zitterbewegung which refers to a highly oscillatory component in the motion of relativistic particles. ${ }^{74}$ Quite generally, such an oscillatory component is always observed when the electron states in two or more neighboring bands interfere. ${ }^{75}$ Of course, such an interference also lies at the heart of (psuedo)spin precession so that the unique transport properties of graphene can be considered to be a manifestation of zitterbewegung in a solidstate system. ${ }^{43}$

The outline of the paper is as follows. In Sec. II we derive a kinetic equation for the density matrix specific to graphene, taking the quantum Liouville equation as our starting point and focusing on the form of the scattering term, which is different in graphene from that in other materials even in the first Born approximation. Fol- 
lowing that, in Sec. III we consider the dynamics of the pseudospin. We divide the pseudospin density matrix into a part representing conserved pseudospin and a part representing non-conserved pseudospin, and demonstrate the way scattering affects both of these parts and connects one to the other. In Sec. IV we determine the steady-state solution in the presence of an electric field and the electrical conductivity. We show that the ordinary, $\tau$-dependent conductivity can be traced to the conserved pseudospin distribution, while the number density and $\tau$-independent conductivity is associated with the non-conserved pseudospin distribution. These arguments are further developed in Sec. V. We illustrate in Sec. VI the remarkable similarities between charge transport in graphene and spin transport in semiconductors with strong spin-orbit interactions, including the way the vertex correction to spin currents in semiconductors has an analog in graphene that reinforces the $n$ - and $\tau$ independent conductivity in the weak momentum scattering regime. We conclude with a brief summary in Sec. VII.

\section{TIME EVOLUTION OF THE DENSITY MATRIX}

The system is described by a density operator $\hat{\rho}$ which obeys the quantum Liouville equation

$$
\frac{d \hat{\rho}}{d t}+\frac{i}{\hbar}\left[\hat{H}+\hat{H}^{E}+\hat{U}, \hat{\rho}\right]=0
$$

where $\hat{H}$ is the band Hamiltonian, $\hat{H}^{E}$ represents the interaction with external fields, and $\hat{U}$ is the impurity potential. We project the Liouville equation onto a set of time-independent states of definite wave vector $\{|\boldsymbol{k} s\rangle\}$ that are not assumed to be eigenstates of $\hat{H}$. The matrix elements of $\hat{\rho}$ in this basis are written as $\rho_{\boldsymbol{k} \boldsymbol{k}^{\prime}} \equiv \rho_{\boldsymbol{k k}^{\prime}}^{s s^{\prime}}=\left\langle\boldsymbol{k} s|\hat{\rho}| \boldsymbol{k}^{\prime} s^{\prime}\right\rangle$, with corresponding notations for the matrix elements of $\hat{H}, \hat{H}^{E}$ and $\hat{U}$, thus $\rho_{\boldsymbol{k} \boldsymbol{k}^{\prime}}, H_{\boldsymbol{k} \boldsymbol{k}^{\prime}}, H_{\boldsymbol{k} \boldsymbol{k}^{\prime}}^{E}$, and $U_{\boldsymbol{k} \boldsymbol{k}^{\prime}}$ are $2 \times 2$ matrices in the space spanned by the pseudospin $s$. We refer to $\rho_{\boldsymbol{k} \boldsymbol{k}^{\prime}}$ as the (pseudospin) density matrix. Matrix elements of the band Hamiltonian $H_{\boldsymbol{k} \boldsymbol{k}^{\prime}}=H_{\boldsymbol{k}} \delta_{\boldsymbol{k} \boldsymbol{k}^{\prime}}$ and $H_{\boldsymbol{k} \boldsymbol{k}^{\prime}}^{E}=H_{\boldsymbol{k}}^{E} \delta_{\boldsymbol{k} \boldsymbol{k}^{\prime}}$ are diagonal in $\boldsymbol{k}$ but contain off-diagonal terms in the pseudospin indices. Matrix elements of the scattering potential $U_{\boldsymbol{k}^{\prime}}$ are off-diagonal in $\boldsymbol{k}$. (Matrix elements of the form $U_{\boldsymbol{k} \boldsymbol{k}}$ lead to a redefinition of $H_{\boldsymbol{k}}$.) We assume elastic scattering and work in the first Born approximation, in which $U_{\boldsymbol{k} \boldsymbol{k}^{\prime}}^{s \prime^{\prime}}=U_{\boldsymbol{k} \boldsymbol{k}^{\prime}} \delta_{s s^{\prime}}$. Impurities are assumed uncorrelated and the normalization is such that the configurational average of $\left\langle\boldsymbol{k} s|\hat{U}| \boldsymbol{k}^{\prime} s^{\prime}\right\rangle\left\langle\boldsymbol{k}^{\prime} s^{\prime}|\hat{U}| \boldsymbol{k} s\right\rangle$ is $\left(n_{i}\left|U_{\boldsymbol{k} \boldsymbol{k}^{\prime}}\right|^{2} \delta_{s s^{\prime}}\right) / V$, where $n_{i}$ is the impurity density, $V$ the crystal volume and $U_{\boldsymbol{k} \boldsymbol{k}^{\prime}}$ the matrix element of the potential of a single impurity.

$\rho_{\boldsymbol{k} \boldsymbol{k}^{\prime}}$ is divided into a part diagonal in $\boldsymbol{k}$ and a part offdiagonal in $\boldsymbol{k}$, given by $\rho_{\boldsymbol{k} \boldsymbol{k}^{\prime}}=f_{\boldsymbol{k}} \delta_{\boldsymbol{k} \boldsymbol{k}^{\prime}}+g_{\boldsymbol{k} \boldsymbol{k}^{\prime}}$, where, in $g_{\boldsymbol{k} \boldsymbol{k}^{\prime}}$, it is understood that $\boldsymbol{k} \neq \boldsymbol{k}^{\prime}$. We will be interested primarily in $f_{\boldsymbol{k}}$ since most operators related with steady state processes are diagonal in $\boldsymbol{k}$. The quantum Liouville equation is broken down into

$$
\begin{aligned}
\frac{d f_{\boldsymbol{k}}}{d t}+\frac{i}{\hbar}\left[H_{\boldsymbol{k}}, f_{\boldsymbol{k}}\right] & =-H_{\boldsymbol{k}}^{E}-\frac{i}{\hbar}[\hat{U}, \hat{g}]_{\boldsymbol{k} \boldsymbol{k}}, \\
\frac{d g_{\boldsymbol{k} \boldsymbol{k}^{\prime}}}{d t}+\frac{i}{\hbar}[\hat{H}, \hat{g}]_{\boldsymbol{k} \boldsymbol{k}^{\prime}} & =-\frac{i}{\hbar}[\hat{U}, \hat{f}+\hat{g}]_{\boldsymbol{k} \boldsymbol{k}^{\prime}}
\end{aligned}
$$

The solution to Eq. (2b) to first order in $\hat{U}$ can be written as

$$
g_{\boldsymbol{k} \boldsymbol{k}^{\prime}}=-\left.\frac{i}{\hbar} \int_{0}^{\infty} d t^{\prime} e^{-i \hat{H} t^{\prime} / \hbar}\left[\hat{U}, \hat{f}\left(t-t^{\prime}\right)\right] e^{i \hat{H} t^{\prime} / \hbar}\right|_{\boldsymbol{k} \boldsymbol{k}^{\prime}},
$$

The assumption that $\varepsilon_{F} \tau / \hbar \gg 1$ allows us to expand $\hat{f}\left(t-t^{\prime}\right)$ around $t$ and retain only $\hat{f}(t)$. (Additional terms are of higher order in $\hat{U}$.) The equation for $f_{k}$ is

$$
\begin{gathered}
\frac{d f_{\boldsymbol{k}}}{d t}+\frac{i}{\hbar}\left[H_{\boldsymbol{k}}, f_{\boldsymbol{k}}\right]+\hat{J}\left(f_{\boldsymbol{k}}\right)=-H_{\boldsymbol{k}}^{E} \\
\hat{J}\left(f_{\boldsymbol{k}}\right)=\frac{1}{\hbar^{2}} \int_{0}^{\infty} d t^{\prime}\left[\hat{U}, e^{-i \hat{H} t^{\prime} / \hbar}[\hat{U}, \hat{f}(t)] e^{i \hat{H} t^{\prime} / \hbar}\right]_{k}
\end{gathered}
$$

The integral in Eq. (4b) is performed by inserting a regularizing factor $e^{-\eta t^{\prime}}$ and letting $\eta \rightarrow 0$ in the end. For potentials $\left|U_{\boldsymbol{k} \boldsymbol{k}^{\prime}}\right| \propto \mathbb{1}$ which are scalars in pseudospin space this integral has the form

$$
\hat{J}\left(f_{\boldsymbol{k}}\right)=\frac{n_{i}}{\hbar^{2}} \lim _{\eta \rightarrow 0} \int \frac{d^{2} k^{\prime}}{(2 \pi)^{2}}\left|U_{\boldsymbol{k} \boldsymbol{k}^{\prime}}\right|^{2} \int_{0}^{\infty} d t^{\prime} e^{-\eta t^{\prime}}\left\{e^{-i H_{\boldsymbol{k}^{\prime}} t^{\prime} / \hbar}\left(f_{\boldsymbol{k}}-f_{\boldsymbol{k}}^{\prime}\right) e^{i H_{\boldsymbol{k}} t^{\prime} / \hbar}+e^{-i H_{\boldsymbol{k}} t^{\prime} / \hbar}\left(f_{\boldsymbol{k}}-f_{\boldsymbol{k}}^{\prime}\right) e^{i H_{\boldsymbol{k}^{\prime}} t^{\prime} / \hbar}\right\} .
$$

Equation (4) is a generalization of Fermi's golden rule or, equivalently, a generalization of the first Born approximation to systems, where the orbital motion is coupled with a (pseudo)spin degree of freedom. ${ }^{68-70}$

\section{PSEUDOSPIN DYNAMICS}

In the following we derive a scattering term specific to graphene and an equation describing the time evolution of the pseudospin. The band Hamiltonian for the carri- 
ers in each valley in single-layer graphene at low doping densities is given by $H_{\boldsymbol{k}}=\hbar v \boldsymbol{\sigma} \cdot \boldsymbol{k}$, where the constant $v$ is the Fermi velocity and $\boldsymbol{\sigma}$ is the (two-dimensional) vector of Pauli matrices in pseudospin space. We emphasize that the Hamiltonian does not depend on the true spin of particles, thus the final result will contain a factor of 2 from the sum over the spin. An additional factor of 2 must account for the valley degeneracy. Consequently final results are multiplied by an overall factor of 4 .

The Hamiltonian $H_{k}$ is formally similar to the spinorbit interaction in spin- $1 / 2$ electron systems,${ }^{66}$ except that the spin-orbit interaction is usually accompanied by a kinetic energy term quadratic in $k$, which is typically much larger than it, and has no analog in graphene. We wish to consider briefly this aspect of $H_{\boldsymbol{k}}$ from the point of view of symmetry. The Hamiltonian $H_{k}$ transforms as a dipole in pseudospin space ${ }^{67}$ unlike the Hamiltonians of spin- $1 / 2$ electron systems, which contain both a dipole (the spin-orbit interaction) and a monopole (the scalar kinetic energy). In the absence of scattering the equations of motion for the monopole and the dipole are decoupled ${ }^{67}$ but when scattering is present the time evolution operator in the scattering term (5) in general mixes the monopole and the dipole. In graphene the monopole of the density matrix is equivalent to the scalar part and the dipole is equivalent to the pseudospin part. Interestingly, because the Hamiltonian for graphene only contains the dipole term, scattering cannot mix the scalar and pseudospin parts of the density matrix. We will see in the next paragraph how these symmetry arguments become relevant.

\section{A. Scattering term}

Next we will evaluate the scattering term (5). For this purpose, the density matrix of graphene is written as $f_{\boldsymbol{k}}=n_{\boldsymbol{k}}+S_{\boldsymbol{k}} \equiv n_{\boldsymbol{k}} \mathbb{1}+\frac{1}{2} \boldsymbol{S}_{\boldsymbol{k}} \cdot \boldsymbol{\sigma}$ with a scalar part $n_{\boldsymbol{k}}$ and pseudospin part $S_{\boldsymbol{k}}$. We substitute $H_{\boldsymbol{k}}$ in the time evolution operator in Eq. (5) and carry out the time integration. In this way we obtain expressions for the action of $\hat{J}$ on $n_{k}$ and $S_{k}$, and we find that, as discussed above, this term does not mix $n_{\boldsymbol{k}}$ and $S_{\boldsymbol{k}}$. The explicit expression for $\hat{J}\left(n_{\boldsymbol{k}}\right)$ is not needed in this work and will not be given. The action of the scattering term on $S_{\boldsymbol{k}}$ is

$$
\begin{aligned}
\hat{J}\left(S_{\boldsymbol{k}}\right)= & \frac{k n_{i}}{8 \hbar \pi v} \int_{k^{\prime}=k} d \theta^{\prime}\left|U_{\boldsymbol{k} \boldsymbol{k}^{\prime}}\right|^{2}\left(\boldsymbol{S}_{\boldsymbol{k}}-\boldsymbol{S}_{\boldsymbol{k}^{\prime}}\right) \\
& \times\left[\boldsymbol{\sigma}(1-\cos \gamma)+(\boldsymbol{\sigma} \cdot \hat{\boldsymbol{k}}) \hat{\boldsymbol{k}}^{\prime}+\hat{\boldsymbol{k}}\left(\boldsymbol{\sigma} \cdot \hat{\boldsymbol{k}}^{\prime}\right)\right]
\end{aligned}
$$

In the above $\theta^{\prime}$ is the polar angle for the direction of $\boldsymbol{k}^{\prime}$ and $\gamma=\theta^{\prime}-\theta$ is the angle between $\boldsymbol{k}^{\prime}$ and $\boldsymbol{k}$. We see in Eq. (6) that the scattering term is qualitatively different from the scattering term in spin- $1 / 2$ electron systems, ${ }^{68}$ both in its angular dependence and in not mixing the scalar and pseudospin parts of the density matrix. Aside from the Born approximation, no further approximations were made in deriving Eq. (6). Yet we emphasize that it is essential for this theory to assume weak momentum scattering since there is no equivalent of the scalar kinetic energy term present in semiconductors with spin-orbit interactions, and in order to derive Eq. (4) one must assume $\varepsilon_{F} \tau / \hbar=v k_{F} \tau \gg 1$. The problem is characterized by two time scales, the pseudospin precession frequency $\varepsilon_{F} / \hbar=v k_{F}$ and $\tau$, and in assuming $v k_{F} \tau \gg 1$ we were able to truncate the scattering term at the leading order in the impurity potential.

\section{B. Time evolution of the pseudospin}

We consider in more detail the equation for the pseudospin part of the density matrix $S_{\boldsymbol{k}}$, in the general case in which a nonzero source term exists on the RHS. Such a source term will be present when an external field is acting on the system, and its form can be derived straightforwardly from the quantum Liouville equation. The specific case of an electric field will be discussed in the following section. The kinetic equation for $S_{\boldsymbol{k}}$ in the presence of a source $\Sigma_{\boldsymbol{k}}$ is

$$
\frac{d S_{k}}{d t}+\frac{i}{\hbar}\left[H_{k}, S_{k}\right]+\hat{J}\left(S_{k}\right)=\Sigma_{\boldsymbol{k}}
$$

The structure of this equation is very important. In order to bring it out we decompose $S_{k}$ into two linearly independent parts, $S_{\boldsymbol{k}}=S_{\boldsymbol{k} \|}+S_{\boldsymbol{k} \perp}$. $S_{\boldsymbol{k} \|}$ is, in matrix language, parallel to the Hamiltonian $H_{k}$, while the remainder $S_{\boldsymbol{k} \perp}$ is orthogonal to $H_{\boldsymbol{k}}$. Because $\left[H_{\boldsymbol{k}}, S_{\boldsymbol{k} \|}\right]=0$ the parallel part $S_{\boldsymbol{k} \|}$ does not change in time under the action of the time evolution operator $e^{i H_{k} t / \hbar}$. In other words $S_{\boldsymbol{k} \|}$ represents the fraction of the pseudospin which is conserved, that is, carriers which are either electrons or holes. Conversely, $S_{\boldsymbol{k} \perp}$ represents the fraction of the pseudospin which is not conserved, i.e., it is precessing. This fraction corresponds to carriers which are a continually changing mixture of electrons and holes. The decomposotion reflects the central importancee of electron-hole coherence for the carrier dynamics in graphene. We remark that the decomposition is fully equivalent to the decomposition of carrier dynamics in relativistic quantum mechanics which contains a smooth part and on oscillatory part known as zitterbewegung. ${ }^{74,75}$ In that sense the physics discussed here represents a direct manifestation of zitterbewegung in graphene. ${ }^{43}$

Specifically, the following equations hold for $S_{\boldsymbol{k} \|}$ and $S_{\boldsymbol{k} \perp}$

$$
\begin{aligned}
S_{\boldsymbol{k} \|} & =\left[\frac{\operatorname{tr}\left(S_{\boldsymbol{k}} H_{\boldsymbol{k}}\right)}{\operatorname{tr}\left(H_{\boldsymbol{k}}^{2}\right)}\right] H_{\boldsymbol{k}}, \\
{\left[S_{\boldsymbol{k} \|}, H_{\boldsymbol{k}}\right] } & =0 \\
\operatorname{tr}\left(S_{\boldsymbol{k} \perp} H_{\boldsymbol{k}}\right) & =0
\end{aligned}
$$

where the symbol tr refers to a trace over the pseudospin 
indices only. It is easily seen that

$$
\begin{aligned}
S_{\boldsymbol{k} \|} & =\frac{1}{2}\left(\boldsymbol{S}_{\boldsymbol{k}} \cdot \hat{\boldsymbol{k}}\right)(\boldsymbol{\sigma} \cdot \hat{\boldsymbol{k}}) \equiv \frac{1}{2} s_{\boldsymbol{k} \|} \sigma_{\boldsymbol{k} \|}, \\
S_{\boldsymbol{k} \perp} & =\frac{1}{2}\left(\boldsymbol{S}_{\boldsymbol{k}} \cdot \hat{\boldsymbol{\theta}}\right)(\boldsymbol{\sigma} \cdot \hat{\boldsymbol{\theta}}) \equiv \frac{1}{2} s_{\boldsymbol{k} \perp} \sigma_{\boldsymbol{k} \perp},
\end{aligned}
$$

where $\hat{\boldsymbol{k}}$ and $\hat{\boldsymbol{\theta}}$ are unit vectors along the direction of $\boldsymbol{k}$ and perpendicular to $\boldsymbol{k}$, respectively. We note that any matrix in pseudospin space can be decomposed as in Eq. (9). Therefore, in analogy with this decomposition of the pseudospin part of the density matrix, we also decompose the source term $\Sigma_{k}$ along the same principles. From Eq. (7) we can immediately see that the equations describing the time evolution of $S_{k \|}$ and $S_{k \perp}$ are

$$
\begin{aligned}
\frac{d S_{\boldsymbol{k} \|}}{d t}+P_{\|} \hat{J}\left(S_{\boldsymbol{k}}\right) & =\Sigma_{\boldsymbol{k} \|}, \\
\frac{d S_{\boldsymbol{k} \perp}}{d t}+\frac{i}{\hbar}\left[H_{\boldsymbol{k}}, S_{\boldsymbol{k} \perp}\right]+P_{\perp} \hat{J}\left(S_{\boldsymbol{k}}\right) & =\Sigma_{\boldsymbol{k} \perp},
\end{aligned}
$$

where $P_{\| / \perp} \hat{J}\left(S_{\boldsymbol{k}}\right)$ indicates that the scattering term acts on $S_{\boldsymbol{k}}=S_{\boldsymbol{k} \|}+S_{\boldsymbol{k} \perp}$ and the resulting expression is projected parallel/perpendicular to the Hamiltonian.

A solution for $S_{k}$ can be found most straightforwardly by expanding $S_{\boldsymbol{k} \|}$ and $S_{\boldsymbol{k} \perp}$ in the transition rate $\left|U_{\boldsymbol{k} \boldsymbol{k}^{\prime}}\right|^{2}$, in a manner analogous to that adopted in determining the steady states of spin distributions in systems with spinorbit coupling. We found ${ }^{69}$ that in steady-state problems the density matrix always contains a correction $\propto \tau$ and is thus of order -1 in the transition rate. This tells us that the expansion of $S_{\boldsymbol{k}}$ needs to start at order -1 . Since we are working in the weak momentum scattering limit we truncate this expansion at the next highest order, which is order zero. The source term $\Sigma_{\boldsymbol{k}}$ does not have any dependence on the transition rate and is thus of order zero. Equating terms of the same order in the transition rate in Eq. (10) shows that the expansion of $S_{\boldsymbol{k} \|}$ must start at order -1 , while the expansion of $S_{\boldsymbol{k} \perp}$ must start at order zero. We denote the order -1 in the transition rate by a superscript $(-1)$ with the corresponding notation for order zero. As a result Eqs. (10) in the weak momentum scattering limit simplify to

$$
\begin{aligned}
P_{\|} \hat{J}\left(S_{\boldsymbol{k} \|}^{(-1)}\right) & =\Sigma_{\boldsymbol{k} \|} \\
\frac{d S_{\boldsymbol{k} \perp}^{(0)}}{d t}+\frac{i}{\hbar}\left[H_{\boldsymbol{k}}, S_{\boldsymbol{k} \perp}^{(0)}\right] & =\Sigma_{\boldsymbol{k} \perp}-P_{\perp} \hat{J}\left(S_{\boldsymbol{k} \|}\right) \\
P_{\|} \hat{J}\left(S_{\boldsymbol{k} \|}^{(0)}\right) & =-P_{\|} \hat{J}\left(S_{\boldsymbol{k} \perp}^{(0)}\right) .
\end{aligned}
$$

In Eq. (11a) we have omitted the time-derivative of $S_{\boldsymbol{k} \|}$ for the following reason. In both equations we are looking for the steady state solution, and the equation for $S_{\boldsymbol{k} \|}$ is most easily solved without the time derivative. The equation for $S_{\boldsymbol{k} \perp}$ is most easily solved with the time derivative explicitly taken into account, but the time dependence drops out in the end.

Equation (11) shows that, if the solution is required only to order zero in the transition rate, the scattering term acts only on $S_{\boldsymbol{k} \|}$, the part of the density matrix parallel to the Hamiltonian. Physically, the fact that $S_{\boldsymbol{k} \|}$ starts at a lower order in the transition rate than $S_{\boldsymbol{k} \perp}$ means that scattering processes are more effective at randomizing the pseudospin than at scattering into pseudospin eigenstates.

Finally, we can simplify the scattering term by projecting Eq. (6) onto and perpendicular to the Hamiltonian $H_{\boldsymbol{k}}$. The projections that we will require in this work are

$$
P_{\|} \hat{J}\left(S_{\boldsymbol{k} \|}\right)=\frac{k n_{i}}{8 \hbar \pi v} \int d \theta^{\prime}\left|U_{\boldsymbol{k} \boldsymbol{k}^{\prime}}\right|^{2}\left(s_{\boldsymbol{k} \|}-s_{\boldsymbol{k} \|}^{\prime}\right)(1+\cos \gamma) \sigma_{\boldsymbol{k} \|},
$$

$$
P_{\perp} \hat{J}\left(S_{\boldsymbol{k} \|}\right)=\frac{k n_{i}}{8 \hbar \pi v} \int d \theta^{\prime}\left|U_{\boldsymbol{k} \boldsymbol{k}^{\prime}}\right|^{2}\left(s_{\boldsymbol{k} \|}-s_{\boldsymbol{k} \|}^{\prime}\right) \sin \gamma \sigma_{\boldsymbol{k} \perp},
$$

$P_{\|} \hat{J}\left(S_{\boldsymbol{k} \perp}\right)=\frac{k n_{i}}{8 \hbar \pi v} \int d \theta^{\prime}\left|U_{\boldsymbol{k} \boldsymbol{k}^{\prime}}\right|^{2}\left(s_{\boldsymbol{k} \perp}+s_{\boldsymbol{k}^{\prime} \perp}\right) \sin \gamma \sigma_{\boldsymbol{k} \|}$.

We proceed to determine the concrete form of $\hat{J}\left(S_{\boldsymbol{k} \|}\right)$ for a screened Coulomb potential. The effect of screening in graphene has been evaluated by Ando $^{71}$ among others, who showed that $k_{T F} \propto k_{F}$, where $k_{\mathrm{TF}}$ is the ThomasFermi wave vector. The explicit expression $k_{T F}$ will not be reproduced here, it suffices to bear in mind that the ration $k_{T F} / k_{F}$ is a constant. In two dimensions, the square of the matrix element $U_{\boldsymbol{k}^{\prime}}$ of a screened Coulomb potential between plane waves is

$$
\begin{aligned}
\left|U_{\boldsymbol{k} \boldsymbol{k}^{\prime}}\right|^{2} & =\frac{Z^{2} e^{4}}{\epsilon_{0}^{2} V_{2 \mathrm{D}}^{2}} \frac{1}{4 k^{2} \sin ^{2} \frac{\gamma}{2}+1 / L_{s}^{2}} \\
& \equiv \frac{W}{\sin ^{2}(\gamma / 2)+k_{\mathrm{TF}}^{2} / k_{F}^{2}},
\end{aligned}
$$

where $Z=1$ is the ionic charge, $L_{s}=k_{F} /\left(2 k_{\mathrm{TF}} k\right)$ is the screening length. Substituting this into Eq. (12a) we obtain

$$
\begin{aligned}
P_{\|} \hat{J}\left(S_{\boldsymbol{k} \|}\right) & =\frac{k n_{i} W}{4 \hbar^{2} \pi v} \int d \theta^{\prime} \zeta(\gamma)\left(s_{\boldsymbol{k} \|}-s_{\boldsymbol{k} \|}^{\prime}\right) \sigma_{\boldsymbol{k} \|} \\
\zeta(\gamma) & =\frac{\cos ^{2}(\gamma / 2)}{\sin ^{2}(\gamma / 2)+k_{\mathrm{TF}}^{2} / k_{F}^{2}}
\end{aligned}
$$

with similar results for $P_{\perp} \hat{J}\left(S_{\boldsymbol{k} \|}\right)$. In order to evaluate this expression we expand $\zeta(\gamma)$ in a Fourier series as $\zeta(\gamma)=\sum_{m} \zeta_{m} e^{i m \gamma}$ and remark that $\zeta_{-m}=\zeta_{m}$. In a similar way we expand $s_{\boldsymbol{k} \|}$ as $s_{\boldsymbol{k} \|}=\sum_{m} s_{k \| m} e^{i m \theta}$. This gives for Eq. (14)

$$
P_{\|} \hat{J}\left(S_{\boldsymbol{k} \|}\right)=\frac{k n_{i} W}{2 \hbar^{2} v} \sigma_{\boldsymbol{k} \|} \sum_{m}\left(\zeta_{0}-\zeta_{m}\right) s_{k \| m} e^{i m \theta} .
$$

This is the furthest this equation can be simplified at this stage. We will see below that in the steady state additional simplifications emerge. 


\section{STEADY STATE SOLUTION}

In the following, we assume low fields $\boldsymbol{E}$ and look for a solution to first order in $\boldsymbol{E}$. As shown in Appendix A in the presence of an electric field $\boldsymbol{E}$ the source term $\Sigma_{\boldsymbol{k}}$ in Eq. (7) takes the form

$$
\Sigma_{\boldsymbol{k}}=\frac{e \boldsymbol{E}}{\hbar} \cdot \frac{\partial S_{0}}{\partial \boldsymbol{k}}
$$

Here $S_{0}$ is the pseudospin part of the equilibrium density matrix, i.e.,

$$
S_{0}=\frac{1}{2}\left(f_{0+}-f_{0-}\right) \sigma_{\boldsymbol{k} \|},
$$

where the scalars $f_{0 \pm}=f_{0}( \pm \hbar v k)$, with $f_{0}$ the FermiDirac distribution and $\pm \hbar v k$ the eigenenergies of the graphene Hamiltonian $H_{\boldsymbol{k}}$, thus

$$
f_{0 \pm}=\frac{1}{e^{\beta(\mp \hbar v k-\mu)}+1}
$$

where $\mu$ is the chemical potential. The conserved and non-conserved components of the source term are

$$
\begin{aligned}
\Sigma_{\boldsymbol{k} \|} & =\frac{e \boldsymbol{E} \cdot \hat{\boldsymbol{k}}}{2 \hbar}\left(\frac{\partial f_{0+}}{\partial k}-\frac{\partial f_{0-}}{\partial k}\right) \sigma_{\boldsymbol{k} \|}, \\
\Sigma_{\boldsymbol{k} \perp} & =\frac{e \boldsymbol{E} \cdot \hat{\boldsymbol{\theta}}}{2 \hbar k}\left(f_{0+}-f_{0-}\right) \sigma_{\boldsymbol{k} \perp} .
\end{aligned}
$$

\section{A. $S_{k \|}$ to leading order in scattering}

Using Eqs. (11a) and (15) we can write down the equation for $S_{\boldsymbol{k} \|}^{(-1)}$ and equate the coefficients of $\sigma_{\boldsymbol{k} \|}$, reducing it to an equation for $s_{\boldsymbol{k} \|}^{(-1)}$. It is important to note from Eq. (19a) that the coefficient of $\sigma_{\boldsymbol{k} \|}$ contains only the Fourier components $m= \pm 1$, and therefore the sum in Eq. (15) will only contain $\zeta_{1}$ (besides $\zeta_{0}$ ). This means that we have for $s_{\boldsymbol{k} \|}^{(-1)}$

$$
\frac{s_{\boldsymbol{k} \|}^{(-1)}}{\tau}=\frac{e \boldsymbol{E} \cdot \hat{\boldsymbol{k}}}{2 \hbar}\left(\frac{\partial f_{0+}}{\partial k}-\frac{\partial f_{0-}}{\partial k}\right) .
$$

Here the scattering time $\tau$ is given by

$$
\frac{1}{\tau}=\frac{k n_{i} W}{2 \hbar^{2} v}\left(\zeta_{0}-\zeta_{1}\right)
$$

where the term in brackets can be expressed as

$$
\zeta_{0}-\zeta_{1}=\left(\sqrt{1+\frac{k_{\mathrm{TF}}^{2}}{k_{F}^{2}}}-\frac{k_{\mathrm{TF}}}{k_{F}}\right)^{2} .
$$

Using Eq. (9a) this gives us $S_{\boldsymbol{k} \|}$ as

$$
S_{\boldsymbol{k} \|}^{(-1)}=\frac{\tau e \boldsymbol{E} \cdot \hat{\boldsymbol{k}}}{4 \hbar}\left(\frac{\partial f_{0+}}{\partial k}-\frac{\partial f_{0-}}{\partial k}\right) \sigma_{\boldsymbol{k} \|} .
$$

\section{B. $S_{k \perp}$ to leading order in scattering}

We proceed to determine $S_{\boldsymbol{k} \perp}^{(0)}$. For this purpose we require the term $P_{\perp} \hat{J}\left(S_{\boldsymbol{k} \|}^{(-1)}\right)$ in Eq. (11b). Inserting Eq. (22) into Eq. (12b) gives

$$
P_{\perp} \hat{J}\left(S_{\boldsymbol{k} \|}^{(-1)}\right)=-\frac{e \boldsymbol{E} \cdot \hat{\boldsymbol{\theta}}}{2 \hbar} \frac{\xi_{0}}{\zeta_{0}-\zeta_{1}}\left(\frac{\partial f_{0+}}{\partial k}-\frac{\partial f_{0-}}{\partial k}\right) \sigma_{\boldsymbol{k} \perp},
$$

where the angular integral $\xi_{0}$ is given by

$$
\xi_{0}=\frac{1}{2 \pi} \int d \theta^{\prime} \frac{\sin ^{2} \frac{\gamma}{2} \cos ^{2} \frac{\gamma}{2}}{\sin ^{2} \frac{\gamma}{2}+\frac{k_{\mathrm{TF}}^{2}}{k_{F}^{2}}}=\frac{1}{2}\left(\sqrt{1+\frac{k_{\mathrm{TF}}^{2}}{k_{F}^{2}}}-\frac{k_{\mathrm{TF}}}{k_{F}}\right)^{2}
$$

so that $\xi_{0} /\left(\zeta_{0}-\zeta_{1}\right)=1 / 2$ and Eq. (23) is indeed indpendent of the screening length. Now Eq. (11b) for $S_{\boldsymbol{k} \perp}^{(0)}$ becomes

$$
\frac{d S_{\boldsymbol{k} \perp}^{(0)}}{d t}+\frac{i}{\hbar}\left[H_{\boldsymbol{k}}, S_{\boldsymbol{k} \perp}^{(0)}\right]=\frac{e \boldsymbol{E} \cdot \hat{\boldsymbol{\theta}} \lambda(k)}{2 \hbar} \sigma_{\boldsymbol{k} \perp}
$$

in which we have abbreviated the quantity

$$
\lambda(k)=\frac{1}{k}\left(f_{0+}-f_{0-}\right)+\frac{1}{2}\left(\frac{\partial f_{0+}}{\partial k}-\frac{\partial f_{0-}}{\partial k}\right) .
$$

The solution of this equation is found most easily using the time evolution operator $e^{i H_{k} t / \hbar}$, and we allow the electric field to have a small but finite frequency $\omega$, taking the limit $\omega \rightarrow 0$ at the end,

$$
S_{\boldsymbol{k} \perp}^{(0)}=\frac{e \boldsymbol{E} \cdot \hat{\boldsymbol{\theta}} \lambda(k)}{2 \hbar} \lim _{\eta, \omega \rightarrow 0} \frac{1}{2 i(2 v k-\omega-i \eta)} \sigma_{\boldsymbol{k} \perp}
$$

As discussed above, unlike $S_{\boldsymbol{k} \|}$ given by Eq. (22), the perpendicular part $S_{\boldsymbol{k} \perp}$ of the spin density matrix is independent of the transition rate $\left|U_{\boldsymbol{k} \boldsymbol{k}^{\prime}}\right|^{2}$.

\section{C. $S_{k \|}$ to zeroth order in scattering}

Finally, $S_{\boldsymbol{k} \|}^{(0)}$ is found from Eq. (11c)

$$
P_{\|} \hat{J}\left(S_{\boldsymbol{k} \|}^{(0)}\right)=-P_{\|} \hat{J}\left(S_{\boldsymbol{k} \perp}^{(0)}\right)
$$

Since $S_{\boldsymbol{k} \perp}^{(0)}$ is known, we need to take the expression for $S_{\boldsymbol{k} \perp}^{(0)}$, act on it with the scattering operator and project the resulting expression parallel to $H$. This will then become the source term for $S_{\boldsymbol{k} \|}^{(0)}$. The details of this process are given in Appendix B. The result is

$$
S_{\boldsymbol{k} \|}^{(0)}=\frac{e \boldsymbol{E} \cdot \hat{\boldsymbol{k}} \lambda(k)}{2 \hbar} \lim _{\eta, \omega \rightarrow 0} \frac{1}{2 i(2 v k-\omega-i \eta)} \sigma_{\boldsymbol{k} \|} .
$$

This term is very similar to $S_{\boldsymbol{k} \perp}^{(0)}$ and their averages over directions in momentum space are the same. 


\section{Electrical conductivity}

Using the velocity operator in single-layer graphene, given by $\boldsymbol{v}=v \boldsymbol{\sigma}$, we can finally determine the electrical current. The current operator depends on the pseudospin and, following our reasoning so far, is decomposed into a parallel part $(\|)$ and a perpendicular part $(\perp)$. The expectation value of the current reads

$$
j_{x}=-e \lim _{\eta \rightarrow 0} \int \frac{d^{2} k}{(2 \pi)^{2}}\left(v_{x \boldsymbol{k} \|} s_{\boldsymbol{k} \|}+v_{x \boldsymbol{k} \perp} s_{\boldsymbol{k} \perp}\right) .
$$

We convert the current tensor into the conductivity tensor $\boldsymbol{\sigma}$ using Ohm's law $\boldsymbol{j}=\boldsymbol{\sigma} \boldsymbol{E}$. The tensor $\boldsymbol{\sigma}$ is diagonal, with $\sigma_{x x}=\sigma_{y y}$ and $\sigma_{x y}=0$. The contribution to the conductivity due to $S_{\boldsymbol{k} \|}^{(-1)}$ (per valley and spin) is

$$
\sigma_{x x}^{\text {ord }}=\frac{e^{2}}{4 h} v k_{F} \tau
$$

$\sigma_{x x}^{\text {ord }}$ behaves differently depending on the nature of scatterers in the system. ${ }^{45}$ For long range scatterers $\tau=$ $\left(4 \hbar^{2} \epsilon^{2} v k_{F}\right) /\left(n_{i} Z^{2} e^{4}\right)$, with $Z$ the atomic number and $\epsilon$ the permittivity, thus $\sigma_{x x}^{\text {ord }} \propto\left(n / n_{i}\right)$. Short range scatterers give $\tau=\left(2 \hbar^{2} \epsilon^{2} v k_{T F}^{2}\right) /\left(n_{i} Z^{2} e^{4} k_{F}\right)$, but since in graphene $k_{T F} \propto k_{F}$ as shown by Ando $^{71}$ we still have the relationship $\sigma_{x x}^{\text {ord }} \propto\left(n / n_{i}\right)$.

The contribution due to $S_{k \perp}^{(0)}$ requires a careful evaluation of $\lim _{\eta, \omega \rightarrow 0} \int d k f_{0 \pm} /(2 v k-\omega-i \eta)$. This is performed by replacing first $1 /(2 v k-\omega-i \eta)$ by $i \pi \delta(2 v k-\omega)$ while keeping $\omega \neq 0$, then evaluating the integral using the $\delta$-function. (Otherwise one can obtain a negative conductivity.) We get

$$
\sigma_{x x}^{0 \perp}=\lim _{\omega \rightarrow 0} \frac{\pi e^{2}}{8 h}\left[\frac{1}{1+e^{\beta(\mu+\hbar \omega / 2)}}-\frac{1}{1+e^{\beta(\mu-\hbar \omega / 2)}}\right] .
$$

Note that the limits $\omega \rightarrow 0$ and $\beta=1 /\left(k_{\mathrm{B}} T\right) \rightarrow \infty$ are not equivalent. Results of a similar or identical magnitude have been found before in the absence of disorder and for $n=0$ only. ${ }^{38-44}$ The contribution of $S_{\boldsymbol{k} \|}^{(0)}$ to the conductivity is equal to (per valley and spin) $\sigma_{x x}^{0 \|}=\sigma_{x x}^{0 \perp}$. This result reflects the fact that $S_{\boldsymbol{k} \perp}^{(0)}$ and $S_{\boldsymbol{k} \|}^{(0)}$ have the same angular average in momentum space. Remarkably this holds for a screened Coulomb potential regardless of the screening length.

\section{DISCUSSION}

We would like to dwell on the role of the pseudospin in the electrical conductivity of graphene. In our analysis the pseudospin density matrix $S_{\boldsymbol{k}}$ is quite generally decomposed into a part $S_{\boldsymbol{k} \|}$ representing conserved pseudospin (i.e., carriers which are either electrons or holes) and a part $S_{\boldsymbol{k} \perp}$ representing nonconserved pseudospin (carriers which are a continually changing mixture of electrons and holes). Pseudospin non-conservation, which is crucial in determining the scattering-independent contribution to the conductivity, occurs in graphene due to the mixing of electron and hole states contained in $H_{\boldsymbol{k}}$ (electron-hole coherence). The steady state in graphene therefore involves non-conservation of the pseudospin due to the mixing of electron and hole states present in the Hamiltonian. The derivation shown in the preceding section demonstrates that the conserved pseudospin distribution gives rise to the fraction $\sigma_{x x}^{\text {ord }}$ of the conductivity given by Eq. (30). $\sigma_{x x}^{\text {ord }}$ corresponds to the ordinary electrical ("Boltzmann") conductivity, which in the steady state is proportional to the carrier number density $n$ and the characteristic scattering time $\tau$. The non-conserved pseudospin distribution gives rise to a second contribution to the electrical conductivity, $\sigma_{x x}^{0 \perp}$, which appears to be independent of the carrier number density and scattering time. However, our analysis shows that this is not the complete answer, since scattering between $S_{\boldsymbol{k} \perp}$ and $S_{\boldsymbol{k} \|}$ produces an additional correction of order zero in the scattering potential, $\sigma_{x x}^{0 \|}$ which reinforces $\sigma_{x x}^{0 \perp}$. Scattering from the non-conserved pseudospin distribution into the conserved pseudospin distribution is represented by $P_{\|} \hat{J}\left(S_{\boldsymbol{k} \perp}\right)$ in Eq. (11c). In that sense scattering between the two distributions has a constructive effect, and we emphasize that this reinforcement holds for screened Coulomb scattering, regardless of the screening length (that is, both short-ranged and long-ranged impurity potentials.) Conversely, the additional correction $\sigma_{x x}^{0 \| b}$, which depends on the number density but not on the impurity density, is also a result of scattering. We emphasize that this reinforcement of the $n$ - and $\tau$ independent contribution to the electrical conductivity in graphene in the weak momentum scattering limit was not found before and constitutes the main result of our work. This work demonstrates the unity behind two situations which until now appeared to be two different limits of the problem of determining the electrical conductivity of graphene. To date no approach has been put forward in which the Boltzmann and $n$ - and $\tau$-independent contributions to the conductivity are treated on the same footing.

\section{COMPARISON WITH SYSTEMS WITH SPIN-ORBIT INTERACTIONS}

The derivation of the steady-state density matrix in graphene presented in this work makes evident the many parallels which exist between the establishment of electrical currents in graphene and the establishment of steadystate spin densities and spin currents in semiconductors with strong spin-orbit interactions. In a recent paper 69 we discussed the nature of the steady state in systems with spin-orbit interactions, and showed that it is very different from the steady state established in usual charge systems. This difference is due to the presence of spin 
precession as a result of spin-orbit coupling. In the steady state in spin-orbit systems the spin density matrix is decomposed into a part representing conserved (i.e., not precessing) spin and a part representing precessing spin. The conserved spin distribution is responsible for the establishment of a steady-state spin density, which is proportional to the carrier number density and the characteristic scattering time $\tau$. The precessing spin distribution is responsible for steady state spin currents, which are independent of the scattering time and in two dimensions appear to be independent of the number density. Interestingly, the correction equivalent to $S_{\boldsymbol{k} \|}^{(0)}$ vanishes in spin-orbit systems. Our recent work ${ }^{69}$ demonstrated that scattering from the conserved spin distribution into the precessing spin distribution produces a correction to the precessing spin distribution which in general acts to reduce spin currents, and in certain circumstances causes the spin current to be zero. In that sense scattering between the two distributions also has a destructive effect. Furthermore, our work showed that this cancelation is due to the same physics that produces the vertex correction to the spin conductivity in Green's functions approaches. ${ }^{72}$

Interestingly, the reinforcement of $\sigma_{x x}^{0}$ in graphene happens in a way that is very similar to the vanishing of the spin current mentioned above. In both cases the effect is caused by the term $P_{\|} \hat{J}\left(S_{\boldsymbol{k} \perp}^{(0)}\right)$ appearing in Eq. (11c). Yet this term makes contributions of different signs in the two systems. We remark that Eq. (25) has been solved by applying the time evolution operator. The product $e^{-i H_{\boldsymbol{k}} t / \hbar} \sigma_{\boldsymbol{k} \perp} e^{i H_{\boldsymbol{k}} t / \hbar}$ is made up of two terms which have different dependencies on the angle of the wave vector $\boldsymbol{k}$. The first term is proportional to $\sigma_{\boldsymbol{k} \perp}$ and the second term is proportional to $\left[H_{\boldsymbol{k}}, \sigma_{\boldsymbol{k} \perp}\right]$. The electrical current operator in graphene is isotropic so that only the first term contributes to the conductivity in this material. In contrast, the spin current operator is proportional to $\boldsymbol{k}$. Thus only the second term contributes to the spin current in spin-orbit coupled systems. These results imply that the correction to the spin current is brought about scattering out of $S_{\boldsymbol{k} \|}$ and into $S_{\boldsymbol{k} \perp}$. On the other hand in graphene it is scattering out of $S_{\boldsymbol{k} \perp}$ and into $S_{k \|}$ that gives rise to the correction to electrical conductivity. From the discussion above we expect this reinforcement to appear as a result of the vertex correction to the charge conductivity in graphene if a linear response approach based on Green's functions is used.

In the field of spin transport a simple and elegant argument has been formulated ${ }^{73}$ which explains why the spin current is necessarily zero in certain systems. Briefly, the spin current is proportional to the rate of change of one of the spin components, which must be zero in the steady state. A similar argument cannot be made for charge transport in graphene, where the charge current is proportional to the pseudospin and does not depend on the rate of change of any quantity in the steady state. This is a reassuring observation: if an analogous argu- ment could be made it would imply that the electrical conductivity of graphene vanishes identically, and this is evidently not the case.

Finally, we would like to point out that in spin- $1 / 2$ electron systems with spin-orbit interactions, where the kinetic energy greatly exceeds the spin-orbit splitting, it is customary to treat the spin-orbit interaction as a perturbation. Yet it is interesting to bear in mind that one expects results qualitatively similar to those in graphene for a Rashba-type Hamiltonian with a very large spinorbit constant.

\section{SUMMARY}

We have examined closely the nature of the steady state established in graphene in the presence of an electric field. We have demonstrated that the steady state in this material contains important qualitative differences from the steady state in other known conductors. The principal reason behind this difference is the existence of a pseudospin degree of freedom, which is related to the coupling between electrons and holes contained in the Hamiltonian. In the weak momentum scattering regime there are two contributions to the electrical conductivity in graphene, one linear in the carrier number density and scattering time and one independent of both. These contributions can be identified with pseudospin conservation and non-conservation respectively, and are connected by scattering processes. Scattering between the non-conserved and the conserved pseudospin distributions doubles the contribution to the conductivity independent of $n$ and $\tau$. Moreover, the steady-state density matrix in graphene displays remarkable similarities to the steady-state spin density matrix in systems with spin-orbit interactions. The contribution linear in $n$ and $\tau$ has an analog in the part of the spin density matrix which yields a steady state spin density, while the contribution independent of $n$ and $\tau$ is analogous to the part of the spin density matrix which yields a steady state spin current. The reinforcement of the $n$-and $\tau$-independent part of the conductivity is due to scattering between the conserved and non-conserved pseudospin distributions. This scattering also has an analogy in spin- $1 / 2$ electron systems with spin-orbit interactions linear in $\boldsymbol{k},{ }^{72,73}$ except in those systems scattering between the conserved and non-conserved spin distributions causes the spin-Hall current to vanish.

We are very grateful to Allan MacDonald, Qian Niu, Branislav Nikolić, Horst Störmer, Philip Kim, Shaffique Adam, Björn Trauzettel, Maxim Trushin, Di Xiao, Wang Yao, Wang Kong Tse, Handong Chen, and Sun Wukong for stimulating discussions. The research at Argonne National Laboratory was supported by the US Department of Energy, Office of Science, Office of Basic Energy Sciences, under Contract No. DE-AC02-06CH11357. 
1 A. K. Geim and K. S. Novoselov, Nature Materials 6, 183 (2007).

2 A. H. Castro Neto, F. Guinea, N. M. R. Peres, K. S. Novoselov, and A. K. Geim, arXiv:0709.1163, to appear in Rev. Mod. Phys.

3 M. I. Katsnelson and K. S. Novoselov, Solid State Commun. 143, 3 (2007).

4 J. R. Williams, L. DiCarlo, and C. M. Marcus, Science 317, 638 (2007).

${ }^{5}$ K. I. Bolotin, K. J. Sikes, Z. Jiang, G. Fudenberg, J. Hone, P. Kim, and H. L. Störmer, Solid State Communications 146, 351-355 (2008).

6 X. Wu, M. Sprinkle, X. Li, F. Ming, C. Berger, and W. A. de Heer, Phys. Rev. Lett. 101, 026801 (2008).

7 J. M. Dawlaty, S. Shivaraman, M. Chandrashekhar, F. Rana, and M. G. Spencer, Appl. Phys. Lett. 92, 042116 (2008).

8 R. Danneau, F. Wu, M. F. Craciun, S. Russo, M. Y. Tomi, J. Salmilehto, A. F. Morpurgo, and P. J. Hakonen, Phys. Rev. Lett. 100, 196802 (2008).

${ }^{9}$ L. DiCarlo, J. R. Williams, Y. Zhang, D. T. McClure, and C. M. Marcus, Phys. Rev. Lett. 100, 156801 (2008).

10 J. H. Chen, C. Jang, S. Xiao, M. Ishigami, and M. S. Fuhrer, Nature Nanotechnology 3, 206 - 209 (2008).

11 S. Russo, J. B. Oostinga, D. Wehenkel, H. B. Heersche, S. S. Sobhani, L. M. K. Vandersypen, and A. F. Morpurgo, Phys. Rev. B 77, 085413 (2008).

12 Z. Jiang, Y. Zhang, H. L. Stormer, and P. Kim, Phys. Rev. Lett. 99, 106802 (2007).

13 C.-H. Park, F. Giustino, M. L. Cohen, and S. G. Louie, Phys. Rev. Lett. 99, 086804 (2007).

14 F. Varchon, R. Feng, J. Hass, X. Li, B. Ngoc Nguyen, C. Naud, P. Mallet, J.-Y. Veuillen, C. Berger, E. H. Conrad, and L. Magaud, Phys. Rev. Lett. 99, 126805 (2007).

15 E. G. Mishchenko, Phys. Rev. Lett. 98, 216801 (2007).

16 S. Das Sarma, E. H. Hwang, and W.-K. Tse, Phys. Rev. B 75, 121406 (2007).

17 V. M. Pereira, J. Nilsson, and A. H. Castro Neto, Phys. Rev. Lett. 99, 166802 (2007); V. M. Pereira, F. Guinea, J. M. B. Lopes dos Santos, N. M. R. Peres, and A. H. Castro Neto, Phys. Rev. Lett. 96, 036801 (2006).

18 D. E. Sheehy and J. Schmalian, Phys. Rev. Lett. 99, 226803 (2007).

19 J. H. Bardarson, J. Tworzydo, P. W. Brouwer, and C. W. J. Beenakker Phys. Rev. Lett. 99, 106801 (2007).

20 T. O. Wehling, A. V. Balatsky, M. I. Katsnelson, A. I. Lichtenstein, K. Scharnberg, and R. Wiesendanger, Phys. Rev. B 75, 125425 (2007).

${ }^{21}$ L. Sheng, D. N. Sheng, F. D. M. Haldane, and L. Balents, Phys. Rev. Lett. 99, 196802 (2007).

22 C. Toke, P. E. Lammert, J. K. Jain, and V. H. Crespi, Phys. Rev. B 74, 235417 (2006).

23 V. M. Apalkov and T. Chakraborty, Phys. Rev. Lett. 97, 126801 (2006).

24 O. Vafek, Phys. Rev. Lett. 97, 266406 (2006).

25 D. Huertas-Hernando, F. Guinea, and A. Brataas, Phys. Rev. B 74, 155426 (2006).

26 V. P. Gusynin, S. G. Sharapov, and J. P. Carbotte, Phys. Rev. B 75, 165407 (2007).

27 C. L. Kane and E. J. Mele, Phys. Rev. Lett. 95, 226801 (2005).
28 Z. H. Qiao, J. Wang, Y. D. Wei, and H. Guo, Phys. Rev. Lett. 101, 016804 (2008).

29 Y. Jiang, D.-X. Yao, E. W. Carlson, H.-D. Chen, and J. Hu, Phys. Rev. B 77, 235420 (2008).

${ }^{30}$ L. Brey, H. A. Fertig, S. Das Sarma, Phys. Rev. Lett. 99, 116802 (2007).

31 L. Brey and H. A. Fertig, Phys. Rev. B 76, 205435 (2007).

32 V. V. Cheianov, V. Fal'ko, and B. L. Altshuler, Science 315, 1252 (2007).

33 B. Trauzettel, D. V. Bulaev, D. Loss, and G. Burkard, Nature Phys. 3, 192 (2007).

34 A. Matulis and F. M. Peeters, Phys. Rev. B 75, 125429 (2007).

35 E. Mariani, L. I. Glazman, A. Kamenev, and F. von Oppen, Phys. Rev. B 76, 165402 (2007).

36 L. Fritz, J. Schmalian, M. Mueller, and S. Sachdev, Phys. Rev. B 78, 085416 (2008).

37 T. Stauber, N. M. R. Peres, and F. Guinea, Phys. Rev. B 76, 205423 (2007).

38 E. Fradkin, Phys. Rev.B 33, 3263 (1986).

39 K. Ziegler, Phys. Rev. Lett. 97, 266802 (2006).

${ }^{40}$ K. Ziegler, Phys. Rev. B 75, 233407 (2007).

41 M. Auslender and M. I. Katsnelson, Phys. Rev. B 76, 235425 (2007).

42 A. W. W. Ludwig, M. P. A. Fisher, R. Shankar, and G. Grinstein, Phys. Rev. B 50, 7526 (1994).

43 M. I. Katsnelson, Eur. Phys. J. B 51, 157 (2006).

44 J. Tworzydlo et al, Phys. Rev. Lett. 96, 246802 (2006).

45 M. Trushin and J. Schliemann, Phys. Rev. Lett. 99, 216602 (2007).

46 K. Nomura and A. H. MacDonald, Phys. Rev. Lett. 98, 076602 (2007).

47 I. Herbut, V. Juricic, and O. Vafek, Phys. Rev. Lett. 100, 046403 (2008).

48 S. Adam, E. H. Hwang, V. M. Galitski, and S. Das Sarma, Proc. Natl. Acad. Sci. USA 104, 18392 (2007).

49 E. H. Hwang, S. Adam, and S. Das Sarma, Phys. Rev. Lett. 98, 186806 (2007).

50 Ya. M. Blanter and I. Martin, Phys. Rev. B 76, 155433 (2007).

51 L. P. Zarbo and B. K. Nikolić, Europhys. Lett. 80, 47001 (2007).

52 B. A. Bernevig, T. L. Hughes, H.-D. Chen, C. Wu, S.C. Zhang, Int. Jour. Mod. Phys. B, 20, 3257 (2006).

53 J. Fernandez-Rossier, J. J. Palacios, and L. Brey, Phys. Rev. B 75, 205441 (2007).

54 D. A. Abanin, P. A. Lee, and L. S. Levitov, Solid State Comm. 143, 77 (2007).

55 X.-Z. Yan, Y. Romiah, and C. S. Ting, Phys. Rev. B 77, 125409 (2008).

56 M. Zarea and N. Sandler, Phys. Rev. Lett. 99, 256804 (2007)

57 K. Sasaki, S. Murakami, and R. Saito, Appl. Phys. Lett. 88, 113110 (2006).

58 Y. Barlas, T. Pereg-Barnea, M. Polini, R. Asgari, and A. H. MacDonald, Phys. Rev. Lett 98, 236601(2007).

59 I. L. Aleiner and K. B. Efetov, Phys. Rev. Lett. 97, 236801 (2006).

60 V. V. Cheianov, V. I. Fal'ko, and B. L. Altshuler, and I. L. Aleiner, Phys. Rev. Lett. 99, 176801 (2007).

61 P. M. Ostrovsky, I. V. Gornyi, A. D. Mirlin, 
Phys. Rev. Lett. 98, 256801 (2007).

62 W. Yao, D. Xiao, and Q. Niu, Phys. Rev. B 77, 235406 (2008).

63 D. S. Novikov, Phys. Rev. Lett. 99, 056802 (2007).

${ }^{64}$ D. A. Abanin, P. A. Lee, and L. S. Levitov, Phys. Rev. Lett. 96, 176803 (2006).

${ }^{65}$ E. V. Castro, K. S. Novoselov, S. V. Morozov, N. M. R. Peres, J. M. B. Lopes dos Santos, J. Nilsson, F. Guinea, A. K. Geim, A. H. Castro Neto, Phys. Rev. Lett. 99, 216802 (2007).

66 R. Winkler, Spin-Orbit Coupling Effects in TwoDimensional Electron and Hole Systems (Springer, Berlin, 2003).

67 D. Culcer, C. Lechner, and R. Winkler, Phys. Rev. Lett. 97, 106601 (2006).

68 N. S. Averkiev, L. E. Golub, and M. Willander, J. Phys.: Cond. Mat. 14, R271 (2002).

69 D. Culcer and R. Winkler, Phys. Rev. B. 76, 245322 (2007).

70 E. L. Ivchenko, Y. B. Lyanda Geller, and G. E. Pikus, Sov. Phys. JETP 71, 551 (1990).

71 T. Ando, J. Phys. Soc. Jpn. 75, 074716 (2006).

72 J. Inoue, G. E. W. Bauer, and L. W. Molenkamp, Phys. Rev. B 70, 041303 (2004).

73 O. V. Dimitrova, Phys. Rev. B 71, 245327 (2005).

74 E. Schrödinger, Sitzungsber. Preuss. Akad. Wiss., Phys. Math. Kl. 24, 418 (1930).

75 R. Winkler, U. Zülicke, and J. Bolte, Phys. Rev. B 75, 205314 (2007).

\section{APPENDIX A: STEADY STATE EXPECTATION VALUES}

Our aim is to derive a kinetic equation for the density matrix in the presence of an electric field $\boldsymbol{E}$. In order to evaluate the electric field-induced source term in the kinetic equation we start from the Liouville equation with the perturbing Hamiltonian $H^{E}=e \boldsymbol{E} \cdot \hat{\boldsymbol{r}}$. The source term in the Liouville equation is

$$
\hat{\Sigma}=-\frac{i e \boldsymbol{E}}{\hbar} \cdot\left[\hat{\boldsymbol{r}}, \hat{\rho}_{0}\right]
$$

where $\hat{\rho}_{0}$ is the density operator in equilibrium, that is, in the absence of the external field. We are interested in the expectation value of an operator $\hat{O}$ (in our case the electrical current), which is found by taking the trace of this operator with the density matrix, and thus with the correction to the density matrix due to the electric field. This correction evidently depends on the source so that we consider first the trace of the operator $\hat{O}$ with the source term due to the electric field. This trace (denoted by $\mathrm{Tr}$ ) is evaluated as follows

$$
\operatorname{Tr}(\hat{O} \hat{\Sigma})=-\frac{i e \boldsymbol{E}}{\hbar} \cdot \int d^{d} r \operatorname{tr} O(\boldsymbol{r})\left[\boldsymbol{r}, \rho_{0}(\boldsymbol{r})\right] .
$$

We express $\rho_{0}(\boldsymbol{r})$ in terms of its Fourier transform, using the convention $\langle\boldsymbol{r} \mid \boldsymbol{k}\rangle=e^{-i \boldsymbol{k} \cdot \boldsymbol{r}}$, as

$$
\rho_{0}(\boldsymbol{r})=\int \frac{d^{d} k}{(2 \pi)^{d}} \int \frac{d^{d} k^{\prime}}{(2 \pi)^{d}} e^{-i\left(\boldsymbol{k}-\boldsymbol{k}^{\prime}\right) \cdot \boldsymbol{r}} \rho_{0}\left(\boldsymbol{k}, \boldsymbol{k}^{\prime}\right),
$$

where $d=2$ for graphene. At this point we substitute the expression for the spatially-inhomogeneous electric field and focus on the part of the density matrix diagonal in wave vector

$$
\operatorname{Tr}(\hat{O} \hat{\Sigma})=\operatorname{tr} \int \frac{d^{d} k}{(2 \pi)^{d}} O(\boldsymbol{k}) \frac{e \boldsymbol{E}}{\hbar} \cdot \frac{\partial \rho_{0}}{\partial \boldsymbol{k}}
$$

This tells us that the source term in the kinetic equation is therefore $(e \boldsymbol{E} / \hbar) \cdot\left(\partial \rho_{0} / \partial \boldsymbol{k}\right)$.

\section{APPENDIX B: $S_{k \|}$ TO ZEROTH ORDER IN SCATTERING}

We will discuss in this section the details of the contribution $S_{\boldsymbol{k} \|}^{(0)}$, which is found from

$$
P_{\|} \hat{J}\left(S_{\boldsymbol{k} \|}^{(0)}\right)=-P_{\|} \hat{J}\left(S_{\boldsymbol{k} \perp}^{(0)}\right)
$$

As mentioned in the main text, $S_{\boldsymbol{k} \perp}^{(0)}$ is known. We act on it with the scattering operator and project the resulting expression parallel to $H$, yielding

$$
\begin{aligned}
P_{\|} \hat{J}\left(S_{\boldsymbol{k} \perp}\right)= & \frac{k n_{i} \lambda(k)}{8 \hbar \pi v} \lim _{\eta, \omega \rightarrow 0} \frac{1}{2 i(2 v k-\omega-i \eta)} \\
& \times \frac{e \boldsymbol{E}}{2 \hbar} \cdot \int d \theta^{\prime}\left|U_{\boldsymbol{k} \boldsymbol{k}^{\prime}}\right|^{2}\left(\hat{\boldsymbol{\theta}}+\hat{\boldsymbol{\theta}}^{\prime}\right) \sin \gamma \sigma_{\boldsymbol{k} \|}
\end{aligned}
$$

The term $\propto \hat{\boldsymbol{\theta}}$ vanishes in the angular integration, while the remaining term gives

$$
P_{\|} \hat{J}\left(S_{\boldsymbol{k} \perp}\right)=-\frac{e \boldsymbol{E} \cdot \hat{\boldsymbol{k}} \lambda(k)}{2 \hbar \tau} \lim _{\eta, \omega \rightarrow 0} \frac{1}{2 i(2 v k-\omega-i \eta)} \sigma_{\boldsymbol{k} \|}
$$

This will then act as the source term for $S_{\boldsymbol{k} \|}^{(0)}$, and the equation is solved in the same way as in Sec. IV A

$$
S_{\boldsymbol{k} \|}^{(0)}=\frac{e \boldsymbol{E} \cdot \hat{\boldsymbol{k}} \lambda(k)}{2 \hbar} \lim _{\eta, \omega \rightarrow 0} \frac{1}{2 i(2 v k-\omega-i \eta)} \sigma_{\boldsymbol{k} \|}
$$

